
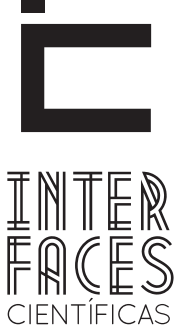

SAÚDE E AMBIENTE

ISSN IMPRESSO 2316-3313

ISSN ELETRÔNICO 2316-3798

DOI -10.17564/2316-3798.2015v4n1p63-69

\title{
ANÁLISE DA QUALIDADE DE VIDA SEGUNDO O QUESTIONÁRIO SF-36 DE PACIENTES SOROPOSITIVAS E SORONEGATIVAS EM DOIS AMBULATÓRIOS DO MUNICÍPIO DE ARACAJU
}

Fernando Henrique Motta Garcia ${ }^{1}$

Mauro Muniz Bezerra ${ }^{3}$
Mário Augusto Ferreira Cruz ${ }^{2}$

Josilda Ferreira Cruz ${ }^{4}$

\section{RESUMO}

A utilização de terapia antiretroviral eficaz para os pacientes HIV-positivos atrasou o aparecimento da AIDS e aumentou a expectativa de vida e o bem-estar dessas pessoas. Apesar disso, os pacientes soropositivos ainda experimentam estresse psicológico resultante do medo da AIDS, e ao estigma associado à doença, bem como discriminação, o que acaba afetando as diferentes dimensões da sua saúde e qualidade de vida. Desse modo, o estudo objetivou comparar a qualidade de vida de pacientes soropositivas com a de pacientes soronegativas. Trata-se de um estudo de corte transversal realizado em dois ambulatórios públicos do município de Aracaju, que contou com 30 pacientes soropositivas e 30 pacientes soronegativas com idade entre 18 e 50 anos. Foi aplicado o questionário SF-36 e questionário sociodemográfico. Análise estatística realizada pelo programa SPSS versão 20.0. Foram avaliadas 60 mulheres, sendo 30 soropositivas e 30 soronegativas. A média de idade das soropositivas foi de 32,87 (DP 8,14) e das soronegativas de 34,87 (DP 9,54). Dos 8 domínios analisados pelo SF36 , o grupo das mulheres soropositivas obteve um escore inferior em 5 deles, sendo os piores escores em estado geral e saúde mental, entretanto, essas diferenças não foram estatisticamente significativas. 0 escore médio, considerando-se todos os domínios, nas pacientes soropositivas foi de 64,82 , e nas soronegativas 69,85 , mas não obteve significância estatística $(p=0,379)$. 0 estudo encontrou uma média geral dos domínios superior a outros estudos e não apresentou diferença estatisticamente significativa entre a qualidade de vida de mulheres soropositivas e soronegativas.

\section{PALAVRAS CHAVE}

Qualidade de vida, HIV, AIDS 


\section{ABSTRACT}

The use of effective antiretroviral therapy for HIV-positive patients delayed the onset of AIDS and increased life expectancy and well-being of these people. Nevertheless, HIV-positive patients still experience psychological stress resulting from fear of AIDS, and the stigma attached to the disease and discrimination, which ultimately affects the different dimensions of their health and quality of life. Thus, the study sought to compare the quality of life of HIV-positive patients with seronegative patients. It is a cross-sectional study conducted in two public clinics in the city of Aracaju, which included 30 patients seropositive and seronegative 30 patients aged between 18 and 50 years. The SF-36 questionnaire and sociodemographic questionnaire was applied. Statistical analysis was performed using SPSS version 20.0. They evaluated 60 women, 30 HIV positive and 30 HIV-negative.
The average age of HIV-positive was 32.87 (SD 8.14) and seronegative of 34.87 (SD 9.54). Of the 8 domains analyzed by the SF-36, the group of HIV-positive women received a lower score in 5 of them, and the worst scores in general health and mental health, however, these differences were not statistically significant. The average score, considering all areas, in HIV-positive patients was 64.82 , and in seronegative 69.85, but did not reach statistical significance $(\boldsymbol{p}=0.379)$. The study found an overall average of the top areas for further studies and showed no statistically significant difference between the quality of life of HIV-positive and HIV-negative women.

\section{KEYWORDS}

Quality of Life. HIV. AIDS.

\section{RESUMEN}

El uso de la terapia antirretroviral para los pacientes VIH-positivos retrasó la aparición de SIDA, aumentó la expectativa de vida y el bienestar de estas personas. Sin embargo, los pacientes VIH-positivos todavía experimentan estrés psicológico que resulta del miedo a SIDA, y el estigma asociado a la enfermedad, así como la discriminación, que afecta las diferentes dimensiones de su salud y calidad de vida. Así, el objetivo de este estudio fue comparar la calidad de vida de pacientes con VIH frente a de los sin VIH. Fue un estudio transversal realizado en dos clínicas públicas en la ciudad de Aracaju, que incluyó a 30 mujeres VIH-positivo y 30 mujeres VIH-negativo de edades entre 18 y 50 años. Se aplicó el cuestionario SF-36 y un sociodemográfico. Análisis estadístico realizado por el programa SPSS versión 20.0. La edad media de las VIH-positivo fue 32,87 (DP 8,14) y VIH-negativo de 34,87
(DP 9,54). De los 8 dominios analizados por el SF-36, el grupo de mujeres con VIH logra una puntuación inferior a 5 de ellos, siendo las peores puntuaciones en el estado general y salud mental, sin embargo, estas diferencias no fueron estadísticamente significativas. La puntuación media, teniendo en cuenta todos los ámbitos, en las pacientes VIH-positivo fue de 64,82 y 69.85 en las VIH-negativo, pero no tuvo significación estadística $(p=0,379)$. El estudio encontró un promedio general de los dominios superior a otros estudios y no mostró diferencia estadísticamente significativa entre la calidad de vida de las mujeres portadoras y no portadoras de VIH.

\section{PALABRAS CLAVE}

Calidad de vida, VIH, SIDA 


\section{INTRODUÇ̃̃̃O}

A AIDS, sigla da denominação original da língua inglesa Acquired Immunodeficiency Syndrome (Síndrome da Imunodeficiência Adquirida) é uma doença causada pelo vírus da imunodeficiência humana (HIV, Human Immunodeficiency Virus), identificado em 1983 (CUNHA; GALVÃO, 2010). A síndrome foi descrita pela primeira vez nos Estados Unidos em 1981, em homossexuais do sexo masculino, anteriormente hígidos que apresentaram pneumonia por Pneumocystis jiroveci e sarcoma de Kaposi. Logo após sua identificação, a infecção pelo HIV rapidamente assumiu caráter de pandemia (LOPES, 2009; GIRONDI ET AL., 2012).

De acordo com o relatório global do Joint United Nations Programme on HIV/AIDS (UNAIDS) realizado em 2013, cerca de 35.3 (32.2-38.8) milhões de pessoas viviam com o vírus da imunodeficiência humana (HIV) em todo o mundo até o ano de 2012, sendo que dessas, 2,3 (1,9-2,7) milhões adquiriram o vírus nesse ano, mostrando um declínio de $33 \%$ de novas infecções em relação ao ano de 2001. O número de mortes, também, declinou de 2,3 milhões em 2005 para 1,6 milhões no ano de 2012. Esses números mostram que mais pessoas estão recebendo a terapia antiretroviral no mundo (UNAIDS, 2013).

A utilização de terapia antiretroviral eficaz para os pacientes atrasou o aparecimento da AIDS e aumentou a expectativa de vida e o bem-estar das pessoas infectadas pelo HIV (RAVETTI; PEDROSO, 2009; HASELI ET AL., 2014). Apesar disso, os pacientes soropositivos ainda experimentam estresse psicológico resultante do medo da AIDS e ao estigma associado à doença, bem como discriminação (SUN ET AL., 2013).

De forma genérica, a Organização Mundial de Saúde (OMS) define qualidade de vida como a percepção do indivíduo de sua posição na vida, no contexto da cultura e sistemas de valores nos quais ele vive em relação aos seus objetivos, expectativas, padrões e preocupações. Esse é um conceito abrangente e multidimensional que envolve diversos domínios (físico, psicológico, social, ambiental e espiritual) (GASPAR ET AL., 2011).

Existem vários instrumentos que analisam a qualidade de vida, um deles é o the Medical Outcomes Study 36-item Short-Form Health Survey (SF-36). Este foi desenvolvido para ser utilizado em grupos de indivíduos, independente da faixa etária, patologia, tratamento, raça, sexo etc. (FRANCO; BARROS; NOGUEIRA-MARTINS, 2005).

Desse modo, o presente estudo objetivou comparar a qualidade de vida de pacientes soropositivas com a de pacientes soronegativas.

\section{MÉTODOS}

Trata-se de um estudo de corte transversal que foi realizado em dois ambulatórios públicos de ginecologia do município de Aracaju: o Serviço de Atendimento Especializado (SAE), localizado no Centro de Especialidades Médicas de Aracaju (CEMAR), especializado no atendimento a indivíduos com HIV/AIDS; e na Unidade de Saúde da Família Edézio Vieira de Melo. Em cada ambulatório foram entrevistadas 30 mulheres para posterior comparação. As pacientes soronegativas foram escolhidas de forma aleatória na sala de espera do consultório médico.

Os critérios de inclusão englobaram indivíduos do gênero feminino, que tinham idade igual ou superior a 18 anos e igual ou inferior a 50 anos, e que aceitaram participar da pesquisa após terem lido e assinado o Termo de consentimento livre e Esclarecido. Foram excluídas do estudo pessoas incapacitadas de responder ao questionário. 0 estudo teve início após aprovação pelo Comitê de Ética e Pesquisa com seres humanos sob número de parecer 1124955.

Os dados foram obtidos a partir da aplicação do questionário SF-36 e questionário sociodemográfico, sendo armazenados em planilhas no Excel e analisados estatisticamente pelo programa SPSS versão 20.0. Para a análise dos escores do questionário SF-36 foi realizada a ponderação dos dados, onde cada questão recebeu um valor previamente estabelecido, em segui- 
da foram calculados os domínios, chamados de RAW SCALE. O nível de significância (p) comparando-se os dois grupos foi calculado por meio do teste $t$-Student.

\section{RESULTADOS}

Foram avaliadas 60 mulheres, sendo 30 soropositivas e 30 soronegativas. A média de idade das soropositivas foi de 32,87 anos (DP 8,14) e das soronegativas de 34,87 anos (DP 9,54).

As características sociodemográficas da população estudada está apresentada na Tabela 1.

Tabela 1 - Características da população estudada*
Quando as soropositivas foram questionadas sobre o modo de transmissão da infecção, a maioria $(76,6 \%)$ referiu à via sexual. Enquanto que apenas uma $(3,4 \%)$ referiu ser infectada devido à transmissão vertical do vírus. As outras seis mulheres (20\%) não sabiam ou não quiseram responder a pergunta por quaisquer motivos.

Dos oito domínios analisados pelo questionário SF-36, o grupo das mulheres soropositivas obteve um escore inferior em cinco deles, sendo os piores escores nos domínios: estado geral e saúde mental. Entretanto, essas diferenças não foram estatisticamente significativas (Tabela 2).

\begin{tabular}{llll}
\hline Variáveis & $\begin{array}{l}\text { Soropositivas } \\
(\mathbf{n = 3 0 )}\end{array}$ & $\begin{array}{l}\text { Soronegativas } \\
(\mathbf{n = 3 0 )}\end{array}$ & $\begin{array}{l}\text { Geral } \\
(\mathbf{n = 6 0})\end{array}$ \\
\hline Estado civil & $22(73,3)$ & $13(43,3)$ & $35(58,3)$ \\
Solteira & $6(20,0)$ & $12(40,0)$ & $18(30,0)$ \\
Casada & $1(3,3)$ & $2(6,7)$ & $3(5,0)$ \\
União estável & $1(3,3)$ & $2(6,7)$ & $3(5,0)$ \\
Divorciada & $0(0,0)$ & $1(3,3)$ & $1(1,7)$ \\
Viúva & & & \\
Escolaridade & $17(56,7)$ & $4(13,3)$ & $21(35,0)$ \\
Fundamental incompleto & $3(10,0)$ & $1(3,3)$ & $4(6,7)$ \\
Fundamental completo & $2(6,7)$ & $3(10,0)$ & $5(8,3)$ \\
Médio incompleto & $6(20,0)$ & $12(40,0)$ & $18(30,0)$ \\
Médio completo & $0(0,0)$ & $6(20,0)$ & $6(10,0)$ \\
Superior incompleto & $2(6,7)$ & $4(13,3)$ & $6(10,0)$ \\
Superior completo & & & \\
Renda & $11(36,7)$ & $6(20,0)$ & $17(28,3)$ \\
Sem rendimento & $8(26,7)$ & $2(6,7)$ & $10(16,7)$ \\
Até 1 salário mínimo & $9(30,0)$ & $17(56,7)$ & $26(43,3)$ \\
1 a 2 salários mínimos & $2(6,7)$ & $3(10,0)$ & $5(8,3)$ \\
2 a 3 salários mínimos & $0(0,0)$ & $2(6,7)$ & $2(3,3)$ \\
3 a 10 salários mínimos & & & \\
\hline
\end{tabular}

Legenda: * Dados em n (tamanho amostral) e \% (percentual da amostra).

Fonte: Dados da pesquisa. 
Tabela 2 - Características das variáveis que indicam a qualidade de vida

\begin{tabular}{|c|c|c|c|c|c|c|c|}
\hline Domínios & Grupo & $\mathbf{N}$ & Média & DP & Mínimo & Máximo & $\mathbf{P}$ \\
\hline \multirow{2}{*}{ Estado Geral } & Soropositivas & 30 & 56,80 & 28,15 & 0,0 & 100,0 & \\
\hline & Soronegativas & 30 & 62,13 & 20,89 & 10,0 & 92,0 & 0,41 \\
\hline \multirow{2}{*}{$\begin{array}{l}\text { Capacidade } \\
\text { Funcional }\end{array}$} & Soropositivas & 30 & 79,67 & 23,41 & 20,0 & 100,0 & \\
\hline & Soronegativas & 30 & 77,57 & 25,24 & 5,0 & 100,0 & 0,74 \\
\hline \multirow{2}{*}{ Aspectos físicos } & Soropositivas & 30 & 60,00 & 42,85 & 0,0 & 100,0 & \\
\hline & Soronegativas & 30 & 77,50 & 40,12 & 0,0 & 100,0 & 0,11 \\
\hline \multirow{2}{*}{$\begin{array}{l}\text { Aspectos } \\
\text { Emocionais }\end{array}$} & Soropositivas & 30 & 65,54 & 40,58 & 0,0 & 100,0 & \\
\hline & Soronegativas & 30 & 76,66 & 39,30 & 0,0 & 100,0 & 0,29 \\
\hline \multirow{2}{*}{$\begin{array}{l}\text { Aspectos } \\
\text { Sociais }\end{array}$} & Soropositivas & 30 & 72,91 & 31,67 & 0,0 & 100,0 & \\
\hline & Soronegativas & 30 & 77,50 & 30,69 & 0,0 & 100,0 & 0,57 \\
\hline \multirow{2}{*}{ Dor } & Soropositivas & 30 & 66,67 & 32,44 & 10,0 & 100,0 & \\
\hline & Soronegativas & 30 & 65,72 & 25,70 & 22,0 & 100,0 & 0,90 \\
\hline \multirow{2}{*}{ Vitalidade } & Soropositivas & 30 & 60,57 & 23,68 & 0,0 & 100,0 & \\
\hline & Soronegativas & 30 & 58,67 & 16,34 & 30,0 & 90,0 & 0,72 \\
\hline \multirow[t]{2}{*}{ Saúde Mental } & Soropositivas & 30 & 56,40 & 23,07 & 0,0 & 96,0 & \\
\hline & Soronegativas & 30 & 63,07 & 28,06 & 8,0 & 100,0 & 0,32 \\
\hline
\end{tabular}

Legenda: $\mathrm{p}$ : significância pelo teste t-student. $\mathrm{n}$ : tamanho amostral. DP: Desvio-padrão.

Fonte: Dados da pesquisa.

0 escore médio, considerando-se todos os domínios, nas pacientes soropositivas foi de 64,82 , e nas soronegativas 69,85, mas não obteve significância estatística $(p=0,379)$.

\section{DISCUSSÃO}

A AIDS é uma doença crônica transmissível de alta morbimortalidade, entretanto, com a disponibilidade da terapia antirretroviral a sobrevida e qualidade de vida desses pacientes têm aumentado ano após ano (SANTOS ET AL., 2012).

O estudo de Brito e outros autores (2014), realizado com 1202 indivíduos soropositivos no estado de Sergipe, encontrou como perfil predominante o sexo masculino, entre 21-50 anos de idade, com nível de instrução entre ensino básico e fundamental e parceiros sexuais fixos/não fixos. Fato que foi coincidente com o encontrado em estudos anteriores (SANTOS, ET AL., 2009; PIERI; LAURENTI, 2012). O presente estudo, realizado apenas com mulheres, encontrou, entre as soropositivas, um perfil com média de idade de 32,87 anos (DP $8,14)$, com maioria solteira ( $73,3 \%)$, com fundamental incompleto $(56,7 \%)$ e sem rendimento (36,7\%).

Em estudo transversal realizado por Hanseli e outros autores (2012) no Irã, com 129 pacientes soropositivos o domínio que obteve maior escore foi capacidade funcional (58), bem como o presente estudo; e o com menor escore foi aspectos físicos $(33,6)$, o que difere do presente estudo, onde a menor pontuação foi no domínio saúde mental $(56,4)$.

Em relação à média geral dos escores de todos os domínios do questionário de qualidade de vida SF-36, o estudo de Hanseli e outros autores (2012) obteve 
uma média inferior ao do presente estudo, sendo 59,5 e 64,82, respectivamente. A melhor qualidade de vida encontrada em gestantes entrevistadas em um município brasileiro parece evidenciar o sucesso da evolução na terapêutica da síndrome da imunodeficiência adquirida, como também avanços na compreensão da doença pela sociedade, o que torna seus portadores cada vez mais integrados ao meio em que vivem, melhorando assim sua qualidade de vida.

Em estudo chinês de Sun e outros autores (2013) que contou com 744 participantes soropositivos, a maior média dos escores foi observada no domínio capacidade funcional (83), e a menor no estado geral de saúde $(45,2)$. No presente estudo o domínio capacidade funcional, também, obteve a maior pontuação, corroborando o estudo chinês. A média geral dos escores no estudo chinês foi de 64,53 (SUN ET AL., 2013), semelhante ao valor obtido no presente estudo $(64,82)$, mostrando qualidade de vida semelhante entre os soropositivos, dentre os dois locais.

Um estudo espanhol que avaliou a qualidade de vida de pacientes soropositivos, usando como grupo controle indivíduos com doença hepática crônica viral, evidenciou que os pacientes soropositivos tiveram escores inferiores nos 8 domínios do SF-36 em comparação com os controles (ORDÓÑEZ ET AL., 2005). A média geral dos escores das pacientes soropositivas foi 58,23 , valor inferior ao obtido no presente estudo $(64,82)$. Já a média geral dos controles do sexo feminino foi 76,26 , valor superior ao obtido nos controles do presente estudo $(69,85)$.

Além da AIDS, outras doenças crônicas podem afetar negativamente na qualidade de vida dos portadores, estando as doenças cardíacas como uma das principais causas. Christmann, Costa \& Moussalle (2011) encontraram uma baixa qualidade de vida entre pacientes com cardiopatia (média geral dos escores 40,02), tendo como menor média o domínio limitação por aspectos físicos, com média de 24,11 . Desse modo, comparando ao presente estudo, os pacientes com cardiopatia de um modo geral podem apresentar uma qualidade de vida inferior aos pacientes soropositivos.

\section{CONCLUSÃO}

Dos oito domínios analisados pelo questionário SF-36, o grupo das mulheres soropositivas obteve um escore inferior em cinco deles, sendo os piores escores, nos domínios: estado geral e saúde mental. Entretanto, essas diferenças não foram estatisticamente significativas. Além disso, o presente estudo encontrou uma média geral dos domínios superior a outros estudos, o que pode refletir a evolução na terapêutica dos portadores de HIV. Estudos com maior amostra são necessários para melhor avaliar o tema.

\section{REFERÊNCIAS}

BRITO, F. G. et al. Perfil epidemiológico de portadores do vírus da imunodeficiência humana e síndrome da imunodeficiência adquirida no estado de Sergipe, 2007-2012. Interfaces Científicas - Saúde e Ambiente, v.2, n.2, 2014. p.59-71.

CHRISTMANN, M.; COSTA, C. C.; MOUSSALLE, L. D. Avaliação da qualidade de vida de pacientes cardiopatas internados em um hospital público. Revista da AMRIGS, v.55, n.3, 2011. p.239-43.

CUNHA, G. H.; GALVÃO, M. T. G. Diagnósticos de Enfermagem em Pacientes com o Vírus da Imunodeficiência Humana/Síndrome da Imunodeficiência Adquirida em Assistência Ambulatorial. Acta Paul

Enferm, v.23, n.4, 2010. p.526-532.

FRANCO, G. P.; BARROS, A. L. B. L.; NOGUEIRA-MARTINS, L. A. Qualidade de vida e sintomas depressivos em residentes de enfermagem. Rev. Latino-Am.

Enfermagem [on-line], v.13, n.2, 2005. p.139-144.

GASPAR, J. et al. Qualidade de vida de mulheres vivendo com o HIV/aids de um município do interior paulista. Rev. esc. enferm. USP [on-line], v.45, n.1, 2011. p.230-236. 
GIRONDI, J. B. R. et al. Perfil Epidemiológico de Idosos Brasileiros que Morreram por Síndrome de Imunodeficiência Adquirida entre 1996 e 2007 . Acta Paul Enferm, v.25, n.2, 2012. p.302-307.

HASELI, N. et al. Health-related quality of life and its related factors in HIV+ patient's referred to Shiraz Behavioral Counseling Center. Med J Islam Repub, v.28, n.13, 2012.

JOINT UNITED NATIONS PROGRAMME ON HIV/AIDS (UNAIDS). Global report: UNAIDS report on the global AIDS epidemic, 2013.

LOPES, A. C. Tratado de clínica médica. 2.ed. São Paulo: Roca, 2009.

ORDÓÑEZ, M. A. G. et al. Calidad de vida relacionada con la salud de Pacientes infectados por VIH medida por el Cuestionario de Salud SF-36. An Med Interna Madrid, v.18, n.2, 2001. p.74-79.

PIERI, F. M.; LAURENTI, R. HIV/AIDS: Perfil Epidemiológico de Adultos Internados em Hospital Universitário. Cienc. Cuid. saude, v.11, 2012. p.144-152.
RAVETTI, C. G.; PEDROSO, E. R. P. Estudo das Características Epidemiológicas e Clínicas de Pacientes Portadores do Vírus da Imunodeficiência Humana em Pronto Atendimento do Hospital das Clínicas da Universidade Federal de Minas Gerais. Revista da Sociedade Brasileira de Medicina Tropical, v.42, n.2, 2009. p.114-118.

SANTOS, M. L. S. G. et al. A Dimensão Epidemiológica da Coinfecção TB/HIV. Rev. Latinoam Enfermagem, v.17, n.5, 2009. p.683-688.

SANTOS, W. S. et al. Gravidez e a Maternidade na Vida de Mulheres Após o Diagnóstico do HIV/ AIDS. Cienc Cuid Saúde, v.11, n.2, 2012. p.250-258.

SUN, W.; WU, M.; QU, P.; LU, C.; WANG, L. Quality of Life of People Living with HIV/AIDS under the New Epidemic Characteristics in China and the Associated Factors. PLoS ONE, v.8, n.5, 2013. 